\title{
Innovaciones curriculares en la formación universitaria de trabajadores sociales
}

\author{
Susana Aurelia Preciado Jiménez ${ }^{1}$ \\ Elba Covarrubias Ortiz ${ }^{2}$ \\ Claudia Angélica Alcaraz Munguía ${ }^{3}$ \\ Mireya Patricia Arias Soto 4
}

JIMÉNEZ, S. A. P. et al. Innovations in the education of social workers: an investigation concerning the impact of changes in the curriculum that attempt to incorporate new models of teaching and learning, Interface Comunic., Saúde, Educ., v.8, n.15, p.303-12, mar/ago 2004.

This article investigates pedagogical innovations introduced when the curriculum of the School of Social Work at the University of Colima was restructured. The relevance of considering social workers, throughout their education, as social actors who partake in the resolution of societal problems is emphasized. The faculty of the School of Social Work analyzed the process of implementation of the new model of education centered on the student from 2001 to 2005 and its results. The results corroborate the importance of the new models of education introduced and indicate some demands that must be met in order to consolidate curricular innovations.

KEY WORDS: Higher education; curriculum; teaching methodology; innovation; social workers; credentialing.

En este artículo se presentan y analisan los dados de los avances de las innovaciones curriculares incluidas en la reestructuración del currículo de la Facultad de Trabajo Social de la Universidad de Colima. Un aspecto importante que se busca en la formación de trabajadores sociales es que éstos sean vistos como actores sociales que participan en la resolución de las necesidades de la sociedad. Para ello, el cuerpo académico de la facultad se ha dado a la tarea de analizar el proceso y los resultados de la implementación del modelo de enseñanza centrado en el aprendizaje en la Facultad de Trabajo Social que se puso en marcha en la generación 2001-2005, para sustentar la reestructuración del plan de estudios. Los resultados apuntan la pertinencia de las nuevas modalidades pedagogicas e algunas consideraciones para sustentación de las innovaciones curriculares implementadas.

PALABRAS CLAVE: Enseñanza superior; curriculum; modalidades pedagógicas; inovación; trabajadores sociales; habilitación profesional.

${ }^{1}$ Profesora investigadora, Facultad de Trabajo Social, Universidad de Colima, México. <preciado@ucol.mx>

2 Profesora investigadora, Facultad de Trabajo Social, Universidad de Colima, México. <coelba@ucol.mx>

${ }^{3}$ Profesora investigadora, Facultad de Trabajo Social, Universidad de Colima, México. <claudian@ucol.mx>

${ }^{4}$ Profesora investigadora, Facultad de Trabajo Social, Universidad de Colima, México. <tsocial@ucol.mx>

${ }^{1}$ Universidad de Colima

Facultad de Trabajo Social

Av. Universidad, n.333

Col. Las Viboras CP 28044

Colima, Colima México 


\section{Introducción}

La educación superior cada día se encuentra frente a una búsqueda constante de modelos de enseñanza-aprendizaje que responda a las necesidades del mundo en el que vivimos; acordes a la modalidad de formar profesionistas con la calidad científica y humanística que exigen las necesidades emergentes, la Facultad de Trabajo Social de la Universidad de Colima, se ha dado a la tarea de realizar investigación sobre modelos pedagógicos en donde los estudiantes alcancen conocimientos, desarrollen habilidades y competencias de trabajo colaborativo, responsabilidad de su propio aprendizaje, ser críticos, reflexivos, así como propiciar actitudes que se orienten al desarrollo armónico, a la convivencia solidaria, la promoción de los derechos humanos; en otras palabras, lograr un desarrollo integral.

En este sentido es que la Facultad de Trabajo Social, mediante su cuerpo académico, se ha dado a la tarea de iniciar un proyecto de investigación en donde se busca analizar, desde la perspectiva del Trabajo Social, las nuevas modalidades de enseñanza-aprendizaje, las cuales se sustentan en el constructivismo $y$ en el aprendizaje significativo.

Congruentes con esta premisa, los profesores que participan en este proyecto de investigación se dedican a analizar los cambios y los impactos tanto de tipo cuantitativo como cualitativo que se han observado en los estudiantes vinculados al modelo de enseñanza centrado en el aprendizaje a partir de febrero de 2003.

\section{Planteamiento del problema}

La sociedad de fines del siglo XX e inicios del XXI se ha caracterizado por una serie de transformaciones que han tocado todos los aspectos del ser humano. Los cambios han sido profundos en los ámbitos políticos, culturales, sociales, económicos, y por supuesto en el educativo. Las consecuencias de esta vorágine y situaciones han repercutido en las dinámicas sociales, en donde podemos observar el auge de la aldea global por un lado, mientras que por otro existe un repunte de los regionalismos, así como del racismo y actitudes de intolerancia que han producido guerras devastadoras y conflictos en distintas regiones del planeta.

La Asociación Nacional de Universidades e Instituciones de Educación Superior en México (ANUIES) han declarado que la educación del siglo XXI necesita propiciar profundas transformaciones en la organización y operación de la educación en general, ya que a la par van surgiendo nuevas necesidades y exigencias relativas a las competencias y conocimientos de los hombres $y$ mujeres para insertarse activamente en el mundo laboral, como lo refiere Bruer (1995), cuando señala que los empresarios se lamentan que demasiados aspirantes a los empleos que ofrecen carecen de habilidades de comprensión, comunicación y razonamiento, habilidades que son necesarias en los actuales puestos de trabajo. Incluso requieren de trabajadores que sean críticos y analíticos, capaces de innovar y de resolver problemas, con un buen dominio de habilidades de expresión oral y escrita, y capaces de aprender en el lugar de trabajo. Desean personas que sepan cómo aprender. En otras palabras, que los egresados de las instituciones de educación superior respondan a las nuevas exigencias de la sociedad en la cual se insertan. 
Varias investigaciones indican la existencia de la misma carencia de habilidades en el ingreso de los estudiantes a la enseñanza superior. Los profesores denuncian que muchos jóvenes llegan al campus sin estar preparados para el nivel de exigencia universitaria. Los estudiantes tienen una escritura pobre, carecen de una comprensión razonable de la historia y de la cultura. Muchos de ellos son incapaces de seguir el pensamiento abstracto $y$ carecen de las adecuadas habilidades de razonamiento formal (Nickerson et al. 1985, p.5, apud Bruer, 1995).

Nos queda claro que los problemas y los desafíos de la educación no se dan en un vacío, forman parte de la compleja maraña de la dinámica económica, social, cultural y política de nuestra época, como refiere Núñez Hurtado (2001), no se puede desvincular la problemática educativa de las tendencias de la sociedad circundante.

En este sentido, las exigencias para la educación superior, nos llevan a estar en una constante búsqueda de modelos de enseñanza-aprendizaje que respondan a las necesidades del mundo en el que vivimos.

Acorde con estos fundamentos, en la Facultad de Trabajo Social, se han incorporado elementos que propician la generación de modelos de enseñanza centrados en el aprendizaje, el cual se caracteriza por la incorporación de un conjunto de objetivos, modalidades, recursos y técnicas que se orientan a lograr aprendizajes significativos de los contenidos curriculares $y$ a aprender a aprender, promoviendo la actividad autónoma del alumno. El concepto de aprender a aprender está relacionado con el concepto de potencial de aprendizaje, y consiste en desarrollar las capacidades del individuo, específicamente del estudiante, a través del mejoramiento de técnicas, destrezas, estrategias y habilidades con las cuales busca acceder al conocimiento.

Bajo esta perspectiva, es que los académicos reconocemos la necesidad de replantear el camino, reflexionar sobre ¿cómo estamos formando a los futuros profesionistas de la disciplina?, ¿Serán personas con las habilidades y capacidades suficientes para buscar oportunidades y espacios en el campo laboral $y$ atender las necesidades que la sociedad les demande? Y este es precisamente el reto que la Facultad debe asumir, en la implementación de las innovaciones curriculares para la formación de sus estudiantes, así como el estar pendiente para formular estrategias pedagógicas que permitan integrar un currículo académico que dote a los estudiantes de conocimientos, habilidades y capacidades que les permitan convertirse en actores activos de su proceso educativo, ser concientes de su propio estilo de aprendizaje, así como estar eficazmente aptos para lograr un espacio en el mercado laboral.

\section{Avances}

Los modelos centrados en el aprendizaje y la interacción profesor y estudiante están influidos por los factores cognitivos, sociales y afectivos; tomando en consideración esto, fue que el proceso de innovación curricular condujo a reformular las estrategias pedagógicas que orientaría a contar con un currículo académico integrado, el cual dote a los estudiantes de conocimientos, habilidades y capacidades que les permitan convertirse en actores activos de su proceso educativo, realizar trabajo cooperativo, ser concientes de su propio 
estilo de aprendizaje y responsables de su proceso de aprendizaje entre otros; así el cuerpo académico de la Facultad, se orientó a elegir un modelo de enseñanza centrado en el aprendizaje (MECAFTS ${ }^{5}$ ) partiendo de los elementos y principios generales de la disciplina de Trabajo Social y teniendo como estructura principal, cuatro grandes estrategias o modalidades didácticas que propicien en el estudiante la adquisición de competencias y habilidades necesarias para el ejercicio profesional.

Las modalidades didácticas elegidas fueron: Tutorial, Disciplinaria, Integrativa, y de Crecimiento Personal, las cuales buscan que el estudiante pueda integrar procesos educativos innovados, acordes con los principios propuestos por la UNESCO, en el informe Delors, los cuales se han aplicado en el modelo de enseñanza utilizado en la Facultad de Trabajo Social:

1 Aprender a conocer: en el cual, la institución debe propiciar que los estudiantes conozcan y dominen los instrumentos y métodos del conocimiento, que se vincula con la modalidad tutorial.

2 Aprender a hacer: en este sentido, se busca que los estudiantes se preparen para hacer aportaciones a la sociedad, así como el propiciar las competencias para el trabajo en equipo, colaborativo, lograr desarrollar habilidades, destrezas, ejecutar procedimientos, técnicas, métodos, entre otros; la cual se relaciona con la modalidad disciplinaria e integrativa.

3 Aprender a convivir: aspecto trascendental en la formación de recursos humanos, pues destaca el elemento de la diversidad, bajo este criterio la educación en valores es uno de los principales componentes para propiciar las actitudes que se orientan al desarrollo armónico y pleno de las personas, a la convivencia solidaria; la promoción de los derechos humanos; así como en la erradicación de los llamados antivalores; principio que corresponde con las modalidades integrativa y disciplinaria.

4 Aprender a ser: la que conjunta el "desarrollo total y máximo posible de cada persona. La educación integral de la que se viene hablando desde finales del siglo XIX y comienzos del XX; aquella del pensamiento autónomo" (Delors, 1996), que se asocia con la modalidad de crecimiento personal.

Al tener en cuenta tanto los elementos y principios de la disciplina como los de la UNESCO, se considera que se parte por tanto de un contexto educativo $y$ social que ubica tanto a profesores $y$ alumnos a saber quienes son, hacia donde se dirigen, en la implementación del modelo de aprendizaje.
${ }^{5}$ MECAFTS: modelo de enseñanza centrado en el aprendizaje en la Facultad de Trabajo Social, en España.

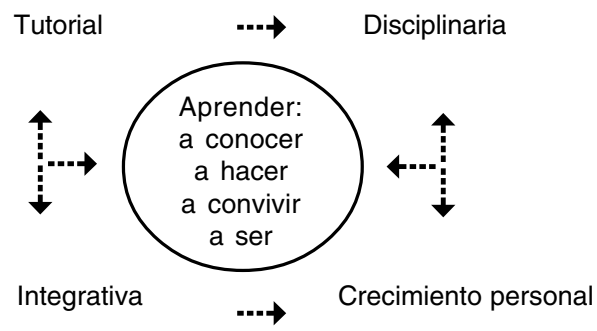


Por tanto, se pretende que con las cuatro modalidades, se propone un diseño holístico dentro del plan curricular, para lo cual se desarrollarán en el estudiante competencias genéricas que le permitirán poseer conocimientos, habilidades y valores, para planear, prevenir, diagnosticar, intervenir, evaluar $e$ investigar en el ámbito de lo social a fin de contribuir al bienestar de individuos y grupos y comunidades, con juicio crítico y compromiso social. Por tanto, la modalidad tutorial, se podría definir como el elemento que busca integrar conocimientos teóricos-conceptuales, la comprensión de los elementos básicos de las diferentes aproximaciones teóricas que permiten a la disciplina del Trabajo Social tener un marco de referencia, así como el dominio de los conceptos metodológicos del Trabajo Social. La modalidad disciplinaria, por otro lado, permite el manejo de fundamentos, procedimientos e instrumentos que permiten al trabajador social resolver demandas planteadas en los campos de aplicación profesional. Las habilidades que se busca desarrollar son que el estudiante pueda aplicar adecuadamente principios y procedimientos requeridos para seleccionar, elaborar y aplicar tácticas que le permitan dar orientación, resolver problemas e interpretar los resultados derivados de su quehacer profesional. Así como la aplicación y comprensión de principios y procedimientos para seleccionar, aplicar $e$ interpretar efectos de estrategias de planeación que promuevan eficazmente la calidad de vida en todos los ámbitos del quehacer profesional del trabajador social $y$ aplicar principios y procedimientos requeridos para comunicar y difundir los resultados y experiencias relevantes de su actividad profesional.

En la modalidad integrativa, se desarrollan habilidades con las cuales el estudiante combine la teoría, con las habilidades técnicas en la práctica profesional. Para ello, se busca que el alumno pueda lograr la integración de casos, dominio y pericia mostrados en la organización de los datos derivados del diagnóstico, evaluación, intervención e investigación para analizar y sintetizar los resultados obtenidos.

$Y$ por último, en la modalidad de crecimiento personal, se busca que el estudiante desarrolle actitudes cooperativas, aprenda a conocerse a sí mismo, a resolver sus problemas personales en los diferentes escenarios; sea consciente de sus capacidades y destrezas, y se proponga metas de acuerdo a sus intereses personales, académicos y profesionales.

\section{El proceso de cambio}

El modelo se ha aplicado actualmente a dos generaciones 2001-2005 y 2002-2006; la generación sujeta de estudio es la 2001-2005, que inició con este modelo a partir del semestre febrero-julio 2003; se seleccionó a ésta por ser la generación de transición en las innovaciones curriculares en la Facultad. El proyecto de innovaciones curriculares, además de buscar la integración del currículo en el cual se conjuga el aprendizaje basado en problemas (ABP), como una de sus estrategias didácticas, las prácticas disciplinarias e integrativas y el crecimiento personal, promueve el desarrollo de destrezas cognitivas de los estudiantes, habilidades profesionales, así como que se desarrollen como personas al ser estudiantes activos y creativos.

Los estudiantes inmersos en este proyecto son alumnos que participan 
dentro de un modelo de aprendizaje en donde el profesor no es el actor principal. Es decir, la relación no se muestra en una sola dirección (Professor $\rightarrow$ Alumno) sino que es compartida y con coparticipación; esta relación se observa directamente en la modalidad tutorial o también denominada estrategia de aprendizaje basado en problemas (ABP).
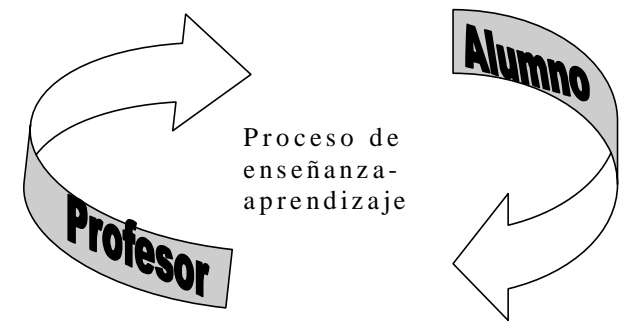

En esta modalidad, el profesor tiene el rol de facilitador, tutor, guía, coaprendiz, mentor o asesor, mientras que los estudiantes asumen un rol de participantes directos, quienes tendrán la responsabilidad de aprender y crear alianzas entre alumno y profesor. Los profesores buscan mejorar la iniciativa de los alumnos y motivarlos. Los alumnos son vistos como sujetos que pueden aprender por cuenta propia. Los alumnos trabajan en equipos para resolver problemas, adquieren y aplican el conocimiento en una variedad de contextos. Los alumnos conformados en pequeños grupos interactúan con los profesores quienes les ofrecen retroalimentación.

Los estudiantes evalúan su propio proceso y el de sus pares. Además el profesor implementa una evaluación integral, en la que es importante tanto el proceso como el resultado.

\section{Metodología}

De acuerdo a las características del estudio, se hizo uso de la metodología cuantitativa y cualitativa. En la primera se utilizaron las técnicas de análisis de documentos de las trayectorias escolares y los resultados educativos, es decir, se revisaron expedientes, calificaciones de los alumnos desde el inicio de sus estudios hasta la fecha. En la segunda se hizo uso de la observación participante, la entrevista exploratoria y el método comparativo. Ser observador participante es facilitado por el hecho de que los investigadores jugamos un papel dentro de los grupos que no altera las pautas usuales de comportamiento de los sujetos observados, en este caso al ser profesorestutores dentro del proceso de enseñanza-aprendizaje nos permite registrar $y$ ser testigos de las situaciones. A través de las entrevistas adquirimos información acerca de las opiniones de todos los actores inmersos en las innovaciones y el método comparativo es de gran utilidad porque nos permite vislumbrar datos significativos de los resultados de generaciones formadas con la metodología tradicional y las formadas con la metodología innovada, así pues la conjugación de estás técnicas nos permitirá realizar una investigación más completa que nos proporcione información eficaz y eficiente para evaluar $y$ mejorar el proceso de cambio. 
El universo de estudio son 171 alumnos que son partícipes en el MECAFTS, la muestra son 45, los cuales fueron elegidos por haber vivido la formación tradicional e innovada dentro de la Facultad, quienes son sujetos a la comparación de sus roles antes y después del MECAFTS. Entendiendo que como parte del proceso de enseñanza-aprendizaje los profesores actores principales, también participarán como sujetos de investigación.

La primera fase del trabajo, que es la que se presenta, es efectuada a partir de la observación que se ha realizado a los sujetos de investigación: alumnos de la generación 2001-2005 y cinco profesores que participaron en los dos momentos de formación (antes y después del MECAFTS); así como entrevistas exploratorias a ambos.

Para determinar los cambios objetivos que se han observado en los estudiantes, se revisaron las trayectorias escolares desde el primero al cuarto semestre, particularmente en las fluctuaciones de los índices de reprobación, retención y deserción escolar.

El análisis se hace a partir de una contrastación entre las actitudes y patrones escolares antes y después del MECAFTS tanto en los alumnos como en los profesores.

\section{Resultados}

Los resultados hasta el momento han sido los siguientes:

En lo que se refiere al aspecto cuantitativo, se han encontrado elementos evidentes en las trayectorias escolares de los estudiantes, en los rubros: aprobación-reprobación, asistencia, promedios y tasa de retención en la generación 2001-2005.

Como se explicó anteriormente, la generación que es objeto de investigación (2001-2005) modificó el modelo de enseñanza tradicional al Modelo de enseñanza centrado en el aprendizaje en el período febrero - julio 2003, la cual cursaba en ese momento el cuarto semestre, las evidencias cuantitativas se observan en los cuadros 1 y 2 .

Cuadro 1 - Promedio de los estudiantes en lo que se refiere a los modelos de enseñanza (2001-2005)

\begin{tabular}{|c|c|c|c|c|}
\hline \multirow{2}{*}{ Generación } & \multicolumn{4}{|c|}{ PROMEDIO } \\
\cline { 2 - 5 } & \multicolumn{3}{|c|}{ Modelo tradicional } & $\begin{array}{c}\text { Modelo centrado } \\
\text { en el aprendizaje }\end{array}$ \\
\cline { 2 - 5 } & $1^{\circ}$ semestre & $2^{\circ}$ semestre & $3^{\circ}$ semestre & $4^{\circ}$ semestre \\
\hline $2001-2005$ & 8.05 & 8.4 & 7.72 & 8.68 \\
\hline
\end{tabular}

Cuadro 2 - Trayectorias escolares de los estudiantes en lo que se refiere a los modelos de enseñanza (2001-2005)

\begin{tabular}{|c|c|c|c|c|c|c|c|}
\hline & & \multicolumn{2}{|c|}{ Aprobados } & \multicolumn{2}{c|}{ Reprobados } & \multicolumn{2}{c|}{ S/ calificacion } \\
\cline { 2 - 8 } Semestre & Alumnos inscritos & $\mathbf{n}-$ & $\%$ & $\mathbf{n}$ & $\%$ & $\mathbf{n}-$ & $\%$ \\
\hline Primero & 69 & 27 & 39.13 & 26 & 37.68 & 16 & 23.18 \\
\hline Segundo & 46 & 28 & 60.86 & 16 & 34.78 & 02 & 4.34 \\
\hline Tercero & 45 & 36 & 80.00 & 06 & 13.33 & 03 & 6.66 \\
\hline Cuarto & 45 & 37 & 82.22 & 08 & 17.77 & - & - \\
\hline
\end{tabular}


En los resultados cualitativos, se observa de igual manera logros en cada una de las modalidades:

Modalidad tutorial: los alumnos afrontan la resolución de problemas planteando objetivos e investigando en diversas fuentes; se presenta el trabajo en equipo y la realización de metas conjuntamente, se da la autoconfianza y autodirección advirtiéndose en su desempeño y seguridad al exponer temas y expresar sus ideas, han desarrollado habilidades para valorar su aprendizaje, aceptación de cambios, relacionan conocimientos previos con nuevos, aprendizaje holístico, en fin se observa una actitud congruente con los principios de la UNESCO.

Modalidad disciplinaria: la modalidad disciplinaria ha permitido la adquisición del manejo de fundamentos, procedimientos e instrumentos propios de la disciplina del trabajo social para que los estudiantes estén preparados a resolver demandas planteadas en los problemas que se revisan en la modalidad tutorial y que en un futuro serán en su ejercicio profesional. Las habilidades que se han podido desarrollar son que el estudiante aplique adecuadamente principios y procedimientos requeridos para seleccionar, elaborar y aplicar conocimientos para resolver situaciones $e$ interpretar los resultados derivados de su quehacer profesional.

Modalidad integrativa: en la modalidad integrativa, se ha logrado que el estudiante pueda combinar la teoría, con las habilidades técnicas en la práctica profesional, que comprenda que los conocimientos, habilidades y capacidades que se desarrollan en las demás modalidades son parte de un todo que finalmente va a redundar en su desempeño.

Modalidad de crecimiento personal: con la modalidad de crecimiento personal se han creado las condiciones para que los estudiantes desarrollen actitudes cooperativas, aprendan a conocerse a sí mismos, a resolver sus problemas personales en los diferentes escenarios; sean conscientes de sus capacidades y destrezas, y se propongan metas de acuerdo a sus intereses personales, académicos y profesionales, se debe admitir que en esta área el trabajo es más complejo, sin embargo, si ha permitido que los alumnos sean concientes de ellos mismos como individuos, que tienen que aprender a vivir $y$ a crecer como personas para llegar a ser unos profesionistas de calidad.

\section{Conclusiones}

Se considera que el modelo de enseñanza centrado en el aprendizaje con sus cuatro modalidades es pertinente para la formación de trabajadores sociales, pues además de propiciar de manera consciente el trabajo colaborativo, permite que los estudiantes compartan la experiencia de aprendizaje, desarrollen habilidades de observación y reflexión, además de favorecer el acercamiento entre profesor-alumnos, alumnos-alumnos.

Coincidimos que la formación de trabajadores sociales en el siglo XXI debe formar actores sociales que participen directamente con la resolución de necesidades de la sociedad, que busque nuevos campos de acción en donde su hacer y que hacer este enfocado al mejoramiento de las condiciones de vida de los pobladores. Por ello es que la Facultad de Trabajo Social busca, a través de la incorporación de innovaciones curriculares, responder a estas 
necesidades y formar profesionales activos, que puedan realizar un trabajo cooperativo, que sean concientes de su realidad social, y propositivos.

El nuevo modelo de enseñanza es un modelo que en la práctica del trabajador social ha sido utilizado, por ello su implementación se considera pertinente. Los beneficios observados en los estudiantes son que éstos han logrado ponerse en contacto con problemas de actualidad, además de desarrollar habilidades que los prepararán para saber enfrentarse a una sociedad cambiante, ser un actor que pueda adaptarse a los escenarios emergentes, y siempre atento a los nuevos temas que surjan. Además de los logros tangibles como son la disminución de la tasa de deserción y reprobación que había sido alta en la Facultad y como resultado de ello un índice de retención de estudiantes más alto, creemos que esto lo podemos tener con el modelo implementado, ya que es una aproximación educacional al proceso del aprendizaje en donde el conocimiento de algo se construye sobre la base del entendimiento previo. También sigue un modelo de red en cuya conformación cada individuo participa activamente, en estrecho contacto con la realidad o el mundo exterior. Aprender a partir de problemas significa hacerlo a partir de la realidad y estimular al estudiante a que construya su propio conocimiento en continuo contacto con el contexto, y esto lo podemos ver actualmente en la Facultad ya que los alumnos investigan la información acerca del problema que se está revisando en tutoría y buscan documentarse con bibliografía pertinente, expertos e Internet lo que permite tener un mejor conocimiento del tema.

Todo ello nos lleva a una reflexión que aprender conlleva la creación de una profesional capaz de establecer relaciones y estar siempre relacionando el objeto de estudio del trabajo social, que es el hombre en su situación social.

Otro elemento importante de resaltar es que las instituciones educativas que se decidan por implementar un modelo centrado en el aprendizaje con modalidad tutorial, deberán estar preparadas con instalaciones e infraestructura adecuadas, personal capacitado y apoyo incondicional de sus autoridades para que ese modelo cuente con un contexto que le permita ser desarrollado en excelentes condiciones y alcance el éxito deseado.

\section{Referências}

BRUER, J. T. Escuelas para pensar: una ciencia del aprendizaje en el aula. Barcelona: Paidós, 1995.

DELORS, J. (Coord.) La educación encierra un tesoro. Informe a la Unesco de la Comisión Internacional sobre la Educación para el siglo XXI presidida por J. Delors. Madrid: Santiliana-Ediciones Unesco, 1997.

NUÑEZ HURTADO, C. Para construir el futuro. In: SIMPOSIUM DE EDUCACIÓN Y VALORES, 7., 2001, México. Anais... México, 2001, p.23-6. 
JIMÉNEZ, S. A. P. et al.

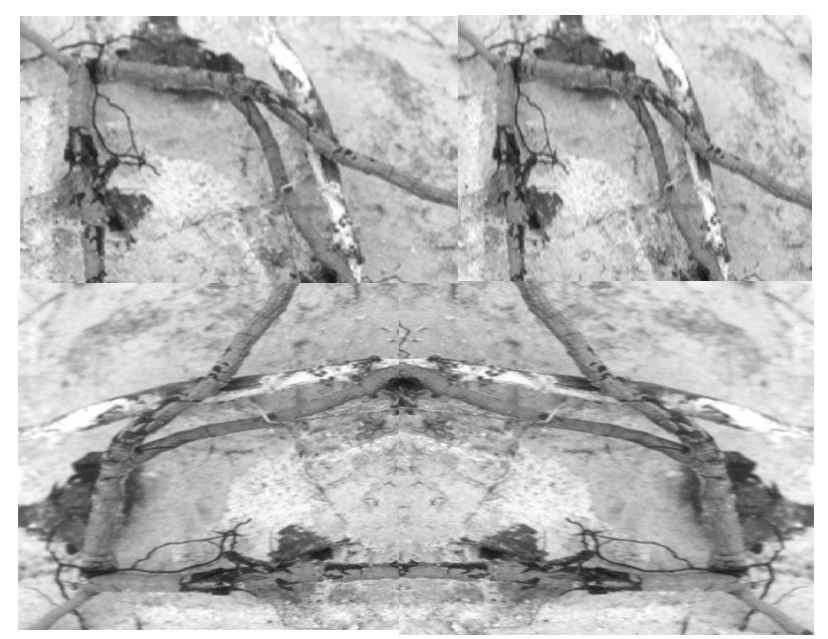

JIMÉNEZ, S. A. P. et al. Inovações curriculares na formação universitária de trabalhadores sociais: uma investigação das mudanças e dos impactos de novos modelos de ensinoaprendizagem, Interface - Comunic., Saúde, Educ., v.8, n.15, p.303-12, mar/ago 2004.

Investigam-se os avanços de inovações incluídas na reestruturação curricular da Faculdade de Serviço Social da Universidade de Colima, a partir da introdução de novas modalidades pedagógicas no currículo. Considera-se a importância de que, na formação de trabalhadores sociais, estes sejam vistos como atores sociais que participam da resolução de problemas da sociedade. Neste sentido, o corpo acadêmico da Faculdade analisou o processo e os resultados da implementação de um modelo de ensino centrado no aluno no período de 2001 a 2005, para sustentar a reestruturação curricular. Resultados obtidos confirmam a pertinência dos modelos adotados e indicam algumas exigências para consolidação das inovações curriculares implementadas.

PALAVRAS-CHAVE: Educação superior; currículo; inovação; metodologia; trabalhadores sociais; formação profissional.

Recebido para publicação em 04/12/03. Aprovado para publicação em 14/07/04. 